\title{
Seudoaneurismas iatrogénicos de arteria femoral: factores de riesgo, prevención y opciones terapéuticas.
}

\author{
Luis Meneses
}

\section{Resumen:}

Los Seudoaneurimas (SA) iatrogénicos de la arteria femoral, se han vuelto más frecuentes debido al aumento en la complejidad de los procedimientos que requieren introductores cada vez de mayor diámetro, asociado a terapias anticoagulantes y/o antiagregantes de largo plazo. El tratamiento estándar de este tipo de lesiones fue por mucho tiempo la cirugía, sin embargo, han aparecido nuevas opciones menos invasivas, como el seguimiento activo, la compresión guiada bajo ultrasonido o la inyec- ción de trombina bajo visión ecográfica. Asimismo, se han estudiado cuales son los factores -ya sea asociados a los pacientes o a las técnicas de punción o hemostasia-, que aumentan las posibilidades de desarrollar SA. Esto ha permitido el desarrollo de dispositivos de tipo "sello arterial" como elementos preventivos.

El presente trabajo tiene por objetivo revisar los factores de riesgo asociados al desarrollo de SA, las medidas de prevención -incluido el uso de "sellos arteriales en ciertos pacientes- , así como las opciones terapéuticas disponibles actualmente para el manejo de pacientes con este tipo de lesiones. 


\section{Introducción:}

Un seudoaneurisma (SA) corresponde a un sangrado continuo desde la arteria femoral puncionada debido a una falla en la hemostasia. Este sagrado se produce al tejido subcutáneo adyacente donde se genera una seudo-cápsula de tejido fibroso, determinando un flujo de entrada y otro de salida al seudoaneurisma, de allí su nombre ya que no posee una pared arterial verdadera ${ }^{1}$. En términos globales la incidencia de SA iatrogénico ha sido reportada entre un 0,05 y un $4 \%$, sin embargo, ésta puede aumentar hasta un $16 \%$ en la medida que se han desarrollado procedimientos más complejos, que requieren accesos cada vez de mayor diámetro y terapia anticoagulante y antiagregante agresivas y de largo plazo ${ }^{2-3}$.

Existen factores de riesgo que aumentan las posibilidades de desarrollarlos, tales como: hipertensión arterial, género femenino, coagulopatía, ateromatosis importante de la arteria femoral común, región inguinal "hostil" (cicatrices, punciones repetidas, presencia de material protésico), bifurcación alta de la arteria femoral, obesidad, cadera rígida, edad avanzada, eritema cutáneo, intertrigo, tratamiento anticoagulante, antiagregante o fibrinolítico ${ }^{4}$.

\section{Diagnóstico Clínico:}

Él cuadro clínico es bastante característico. Existe el antecedente de la realización de un procedimiento endovascular. Habitualmente el paciente debuta con una masa pulsátil, con frémito a la palpación y "soplo" a la auscultación. Es dolorosa y de rápido desarrollo. Sin embargo, ha sido reportada también la existencia de SA "sub-clínicos" que pueden resolver espontáneamente . $^{5}$.

El riesgo de dejar sin tratamiento un SA de la arteria femoral incluye la rotura, la necrosis de la piel adyacente, embolia distal, y síntomas neurológicos debido a compresión local. Existen algunos signos que sugieren la posibilidad de cierre espontáneo como la existencia de baja velocidad de flujo en el estudio Doppler y el tamaño pequeño ${ }^{6}$.

Se han descrito rangos de tiempo muy variable entre el procedimiento endovascular, el diagnóstico y tratamiento del SA de entre 1 día y tres semanas, sin que se afecten los resultados ${ }^{7}$.

\section{Diagnóstico por imagen: Ultrasonido Doppler}

El diagnóstico por imagen del SA se basa en la siguiente combinación de hallazgos al ultrasonido: a) Saco hipoecogénico adyacente a arteria afectada; b) Flujo en "espiral" ("signo del Ying-Yang”, Figura 1) en ecografía Doppler color, éste se produce por la entrada y salida de sangre al saco formado por la seudo-cápsula fibrosa, c) Curva Doppler de "entrada y salida de alta resistencia" al estudiar el cuello SA (Figura 2) ${ }^{10}$ Este hallazgo es muy relevante ya que permite descartar la existencia de una fístula arteriovenosa (FAV) asociada al SA. En caso de existir una FAV el flujo en el cuello será de baja resistencia. La presencia de FAV contraindica la inyección de trombina ${ }^{7}$.

Otros elementos a evaluar al momento del diagnóstico es la morfología del SA. La técnica de tratamiento varía de acuerdo a la cantidad de lóbulos presentes en la lesión ${ }^{7}$.

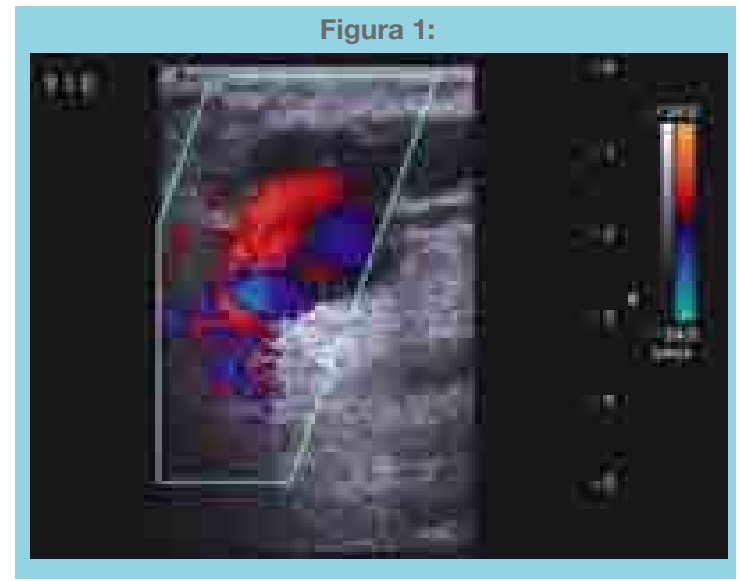

Seudoaneurisma con signo de Ying-Yang, determinado por la señalar color Doppler de entrada (rojo en este caso) y de salida (azul en este caso).

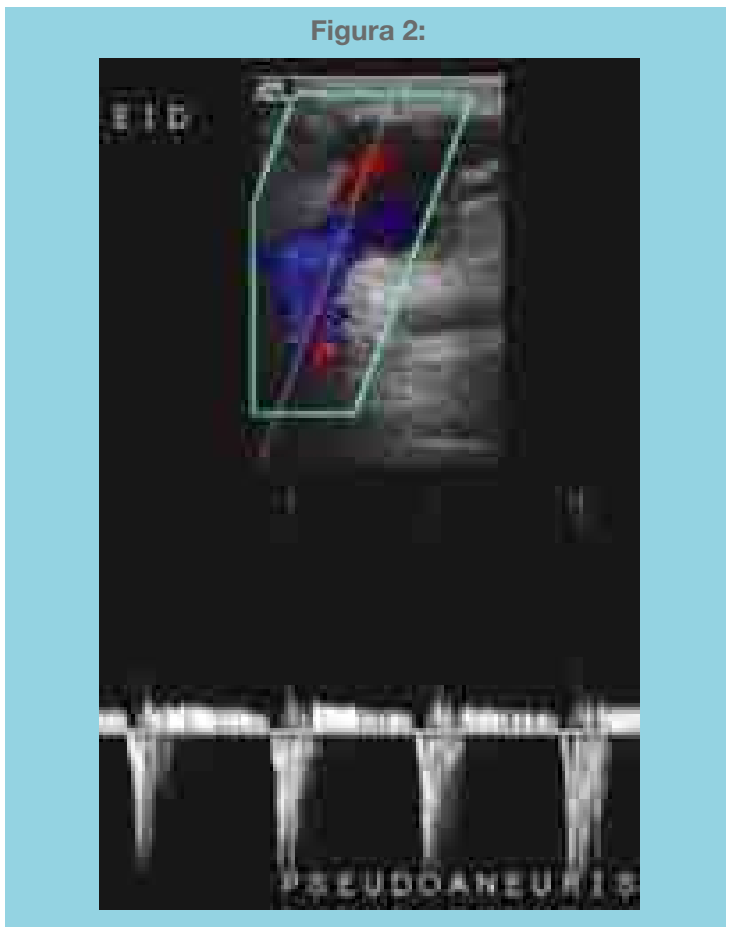

Estudio con curva Doppler de alta resistencia a nivel del cuello lo que descarta la existencia de una fístula arterio-venosa. 


\section{Diagnóstico Diferencial:}

Existen algunas condiciones que clínicamente pueden confundirse con un seudo aneurisma y deben tenerse en consideración al momento de realizar el estudio ecográfico diagnóstico. Entre estos elementos debemos considerar ${ }^{8}$ :

- Linfonodos y adenopatías.

- Fístula arterio venosa.

- Hematoma

- Absceso

-Linfocele

- Trombosis venosa profunda

- Hernia femoral

\section{Opciones terapéuticas:}

En 2009 fue publicado un meta-análisis ${ }^{9}$ acerca del efecto de la compresión y la inyección de trombina guiada por ultrasonido, comparadas con la cirugía como tratamiento del SA iatrogénico de la arteria femoral común. Éste concluyó que la evidencia de alto nivel era escasa al respecto. Sólo logró identificar trabajos randomizados comparando compresión ciega versus compresión guiada bajo ecografía y compresión guiada bajo ecografía versus inyección de trombina guiada por ultrasonido, sin encontrar estudios que incluyeran la cirugía dentro de las opciones de randomización. Los autores concluyen que la evidencia es limitada pero que parece ser adecuado utilizar la inyección de trombina como terapia de elección en pacientes correctamente seleccionados.

En nuestra opinión no existe una opción de tratamiento única para todos los SAs siendo necesaria seleccionar la terapia más adecuada para cada caso en particular.

A continuación detallaremos las opciones terapéuticas más frecuentemente utilizadas en el manejo de éste tipo de lesiones:

\section{1) Manejo conservador}

Los SAs pequeños pueden tener un curso benigno llegando incluso a ocluirse espontáneamente. Por éste motivo, SAs pequeños con escaso flujo o parcialmente trombosados a la ecografía Doppler pueden ser simplemente seguidos con estudios ecográficos seriados reservando otros procedimientos para aquellos que no se resuelvan o progresen en el seguimiento ${ }^{10}$.

Kent et al., demostraron que aquellas lesiones menores a $1,8 \mathrm{~cm}$ habitualmente se trombosaban espontáneamente sin necesidad de ningún procedimiento extra ${ }^{10} \mathrm{en}$ un promedio aproximado de 22 días. Por otra parte Toursarkissian et al. ${ }^{11}$, reportaron que $S A s$ de hasta $3 \mathrm{~cm}$ podían resolver de manera espontánea, sin embargo, en este grupo no se incluyó a pacientes que requería terapia anticoagulante de larga duración, ya que eran tratados de manera quirúrgica, lo que determina un sesgo significativo en la selección.

En general, las recomendaciones sugieren evitar el tratamiento conservador en pacientes con alguna de las siguientes condiciones: lesiones de gran tamaño, necesidad de anticoagulación o antiagregación agresiva y que no es posible suspender, expansión del SA durante el seguimiento, dolor, infección (aneurisma micótico), isquemia distal, preferencia del paciente, imposibilidad de realizar un seguimiento adecuado o infección de la región inguinal.

\section{2) Cirugía}

La cirugía fue considerada la terapia de elección en el manejo de los SAs hasta el año 1991 en que aparecieron los primeros reportes utilizando la compresión guiada por ecografía con el objetivo de trombosar el saco aneurismático ${ }^{12}$.

El éxito de la reparación quirúrgica es cercana al 100\%, sin embargo, la morbilidad puede alcanzar el $25 \%$ con una mortalidad de hasta $3 \%$, esto se debe a que frecuentemente son pacientes con co-morbilidades significativas que en definitiva fueron las causantes del procedimiento inicial, asimismo la existencia de hematomas y la intervención previa predispone a cicatrización inadecuada o infección de la herida operatoria.

Nuestra recomendación es reservar la cirugía para pacientes fuera de criterios para manejo conservador y en que existe contraindicación o falla en la inyección de trombina guiada bajo ultrasonido. En el caso particular de la existencia de FAV asociada al SA puede plantearse la posibilidad de utilizar un stent cubierto para reparar la lesión en pacientes añosos, con factores de riesgo quirúrgico importantes o con una expectativa de vida limitada. En el resto de los casos especialmente en pacientes jóvenes, la cirugía aparece como una opción más adecuada.

\section{3) Compresión guiada por ultrasonido.}

Fellmeth et al., reportó la compresión dirigida por ecografía ${ }^{12}$ como alternativa para el tratamiento de los SAs iatrogénicos postergando a la cirugía como alternativa en caso de falla. Este procedimiento puede ser bastante molesto y doloroso por lo que en muchas ocasiones se requiere sedación además de demandar mucho tiempo. Asimismo, la tasa de falla es muy alta, cercana al $30-40 \%$ en pacientes anticoagulados ${ }^{13}$. 
Consideramos la compresión como una alternativa previa a la cirugía en caso que no exista el recurso técnico de la inyección guiada por ultrasonido.

\section{4) Inyección de trombina:}

La trombina es una enzima que ayuda en la conversión del fibrinógeno a fibrina dentro de la cascada de la coagulación, en sus etapas finales (figura 3). Es por éste motivo que logra evitar el efecto de anticoagulantes como la heparina y la cumadina y estimula mediante su actividad enzimática la formación de coágulo de fibrina en el sitio donde es inyectada al facilitar la conversión de fibrinógeno en fibrina. El primer reporte acerca del uso de la inyección guiada por ultrasonido de trombina en un SA fue en el año 1986 por Cope and Zeit ${ }^{14}$.

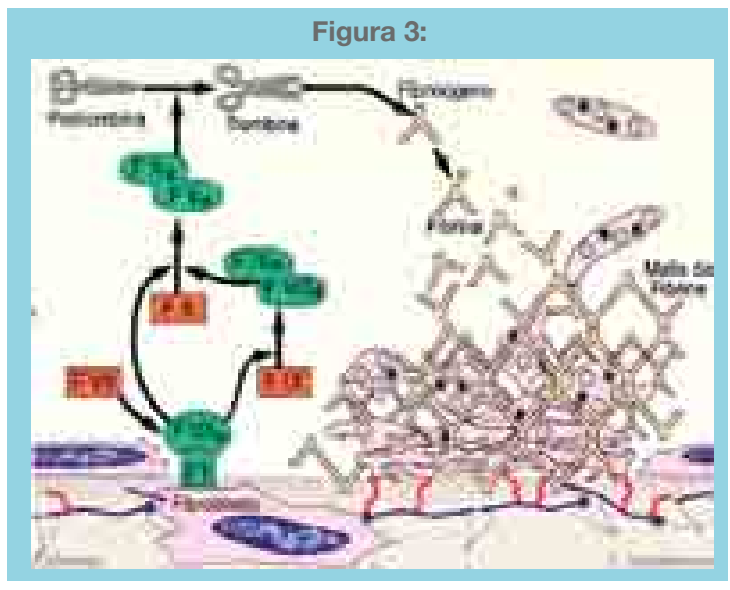

En la cascada de la coagulación la trombina actual en las fases terminales lo que asegura su efectividad incluso en pacientes con anticoagulación cuyo efecto es en etapas previas (Gentileza Dr. Diego Mezzano)

Simplemente, consiste en posicionar una aguja fina en el saco del SA guiándose con ultrasonido, inyectar trombina y estimular la formación de trombo en el interior de la lesión. Es un procedimiento que no requiere preparación ni sedación especial y es mínimamente invasivo ${ }^{15}$. Tampoco requiere interrumpir tratamientos anticoagulantes o antiagregantes.

Las formas de trombina más frecuentemente usadas son la humana y la bovina. Esta última, tiene más posibilidades de generar reacciones alérgicas, que es la complicación más frecuente al utilizar la inyección de trombina como terapia de $\mathrm{SA}^{16}$. Otra opción es la utilización de trombina autóloga, es decir, mediante una muestra de sangre del propio paciente se extrae la trombina necesaria a través de un proceso que puede ser más económico que la compra de kit de trombina humana o bovina comercial ${ }^{17}$.

Consideramos que la inyección debe realizarse lo más cercana posible al cuello del SA, posicionándose en el flujo de entrada y evitando obviamente la inyección en la arteria nativa. La inyección se realiza con aguja de 19 a 25 G utilizando una jeringa de $1 \mathrm{ml}$ de tuberculina.

La dosis reportadas de trombina utilizada varía de 20 a $1000 \mathrm{UI}^{18}$, en nuestra experiencia con dosis entre $150 \mathrm{y}$ 300 UI de trombina se logra una adecuada resolución en la mayoría de los casos.

El éxito del procedimiento ha sido reportado entre un 90$100 \%$ (figura 4$)^{19}$. La recurrencia alcanza hasta un 6\% (19), y si esto ocurre, es posible realizar una nueva inyección u optar por el tratamiento quirúrgico de acuerdo a cada caso en particular. El principal factor asociado a la falla o recurrencia luego de la inyección de trombina es el tamaño y morfología del daño en la pared, en aquellas en que el defecto es de mayor tamaño o es similar a una laceración, la probabilidad de éxito es mucho menor.

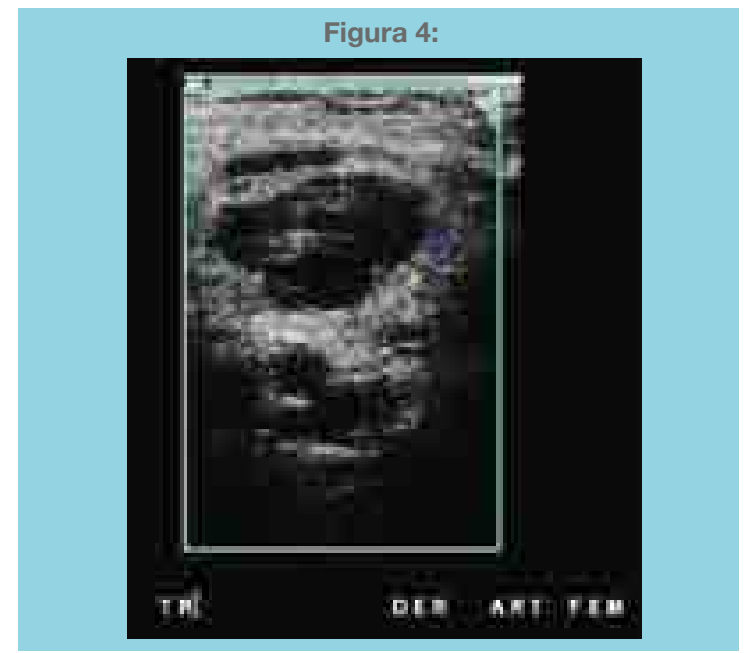

Seudoaneurisma con trombosis completa luego de inyección de trombina. Como se puede apreciar existe material ecogénico que corresponde al trombo formado además de no existir señal de flujo en Doppler color.

El riesgo de complicaciones trombóticas o embólicas es muy bajo y es más bien teórico debido a que la trombina rápidamente es afectada por el efecto de la antitrombina cuando existe paso de la sustancia inyectada al torrente sanguíneo, por lo que el efecto desaparece en segundos. De hecho, el meta-análisis publicado ${ }^{9}$ no reportó ninguna complicación de este tipo en el grupo de pacientes tratados con inyección de trombina.

Si bien la experiencia aún es limitada, ya se han publicado una serie de reportes de uso exitoso de esta técnica en 
SA en otros territorios como en arteria temporal, subclavia, tibial, braquial y radial. Este último es de gran relevancia considerando que es un tipo de acceso vascular que se utiliza en procedimientos endovasculares en forma cada vez más frecuente ${ }^{20}$.

\section{Medidas de prevención:}

Existen trabajos que han demostrado que la punción de la arteria femoral superficial o profunda, en vez de la común para la realización de procedimientos endovasculares, es un factor de riesgo para el desarrollo de SA, por lo que evitar esto es la recomendación ${ }^{21}$.

Por otra parte, la técnica en el retiro de los introductores es muy importante. La manera clásica fue descrita por el Dr. Sven Seldinger en 1950 y consiste en la compresión manual que debe durar 10 o más minutos, con reposo absoluto luego del retiro durante al menos 6 horas ${ }^{22}$.

Comprensiones de menos de 10 minutos han demostrado aumentar el riesgo del desarrollo de Seudo aneurismas ${ }^{23}$.

Los dispositivos de compresión mecánica y sellos arteriales han aparecido como alternativa para disminuir el riesgo del desarrollo de complicaciones locales, sin embargo, usados en población no seleccionada, si bien posibilitan una deambulación precoz, lo que mejora la satisfacción del paciente frente al procedimiento, no han demostrado alterar la incidencia de complicaciones locales ${ }^{24}$ comparado con grupos manejados de manera habitual. El uso de este tipo de dispositivos debe reservarse para situaciones específicas y no aplicarse de manera indiscriminada a población no seleccionada.

\section{Conclusión:}

El manejo de los SAs iatrogénicos de la arteria femoral debe iniciar mediante la prevención, esforzándose en una adecuada técnica de punción y hemostasia, reservando los dispositivos de cierre arterial para casos seleccionados.

Una vez constituida la lesión, en los pacientes sin factores de riesgo y con sacos menores de $1,8 \mathrm{~cm}$, es posible plantear observación seriada con ecografía Doppler. En aquellos en que no es posible plantear manejo conservador la terapia de elección, debiese ser la inyección de trombina dejando la compresión guiada con ultrasonido cuando no exista el recurso técnico. La cirugía aún tiene su uso en los casos refractarios a las terapias guiadas por ecografía o cuando exista asociada fístula arteriovenosa.

\section{Referencias:}

1. OLSEN DM, RODRIGUEZ JA, VRANIC M, RAMAIAH V, RAVI R, DIETHRICH EB, et al. A prospective study of ultrasound injection of femoral pseudoaneurysm: a trend Howard minimal medication. J Vasc Surg 2002; 36: 779 82.

2. KRESOWIK TF, KHOURY MD, MILLER BV, WINNIFORD MD, SHAMMA AR, SHARP WJ, et al. A prospective study of the incidence and natural history of femoral vascular complications after transluminal coronary angioplasty. J Vasc Surg 1991; 13: 328-36.

3. MESSINA LM, BROTHERS TE, WAKEFIELD TW, ZELENOCK GB, LINDENAUER SM, GREENFIELD LJ, et al. Clinical characteristics and surgical Management of vascular complications in patients undergoing cardiac catheterization: interventional versus diagnostic procedures. J Vasc Surg 1991; 13: 593-600.

4. MULLER DWM, SHAMIR KJ, ELLIS SG, TOPOL EJ. Peripheral vascular complications after conventional and complex percutáneos coronary intervention procedures.
Am J Cardiol 1992; 69: 63-68

5. TOURSARKISSIAN B, ALLEN BT, PETRINEC D, THOMPSON RW, RUBIN BG, REILLY JM, et al. Spontaneous closure of selectediatrogenic pseudoaneurysms and arteriovenous fistulae. J Vasc Surg 1997; 25: 803-8.

6. MALEUX G, HENDRICKX S, VANINBROUKX J, LACROIX H, THIJS M, DESMET W, et al. Percutaneous injection of human thrombin to treat iatrogenic femoral pseudoaneurysms: short and midterm ultrasound follow-up. Eur Radiol 2003; 13: 209-12.

7. OLSEN D, RODRÍGUEZ J, VRANIC M, RAMAIAH V, RAVI R, DIETHRICH E. A prospective study of ultrasound scan-guided thrombin injection of femoral pseudoaneurysm: A trend toward minimal medicación. J Vasc Surg 2002; 36: 779-82.

8. MIDDLETON WD, DAYSAM A, TEEFEY S. Diagnosis and treatment of iatrogenic femoral artery pseudoaneurysms. Ultrasound Q 2005; 21: 3-17.

9. TISI PV, CALLAM MJ. Treatment for femoral pseudoaneurysms. Cochrane Database Syst Rev. 2009; 2: CD004981. 
10. KENT KC, MCARDLE CR, KENNEDY B, BAIM DS, ANNINOS E, SKILLMAN JJ. A prospective study of the clinical outcome of femoral pseudoaneurysms and arteriovenous fístulas induced by arterial puncture. J Vasc Surg 1993; 17: 125-33.

11. TOURSARKISSIAN B, ALLEN BT, PETRINEC D, RUBIN BG, REILLY JM, ANDERSON CB, et al. Spontaneous closure of selected iatrogenic pseudoaneurysms and arteriovenous fistulae. J Vasc Surg 1997; 25: 803-809

12. FELLMETH BD, ROBERTS AC, BOOKSTEIN JJ, FREISCHLAG JA, FORSYTHE JR, BUCKNER NK, et al. Postangiographic femoral artery injuries: nonsurgical repair with ultrasound guided compression. Radiology 1991; 178: 671-5.

13. EISENBERG L, PAULSON EK, KLIEWER MA, HUDSON MP, DELONG DM, CAROLL BA. Sonographically guided compression repair of pseudoaneurysms: further experience from a single institution. AJR Am J Roentgenol 1999; 173: 1567-1573

14. COPE C, ZEIT R. Coagulation of aneurysms by direct percutáneos thrombin injection. AJR Am J Roentgenol 1986; 147: 383-7.

15. SULTAN S, NICHOLLS S, MADHAVAN P, COLGAN MP, MOORE D, SHANIK DG. Ultrasound guided human thrombin injection. A new modality in the management of femoral artery pseudoaneurysms Eur J Vasc Endovasc Surg 2001; 22: 542-5.

16. TSETIS D. Endovascular Treatment of Complications of Femoral Arterial

Access. Cardiovasc Intervent Radiol 2010; 33:457-468

17. QUARMBY JW, ENGELKE C, CHITOLIE A, MORGAN RA, BELLI AM. Autologous thrombin for treatment of pseudoaneurysms. Lancet 2002; 359:946-947

18. HANSON JM, ATRI M, POWER N. Ultrasound-guided thrombin injection of iatrogenic Groin pseudoaneurysm: Doppler features and technical tips Br J Radiol 2008; 81: 154-163.

19. OLSEN DM, RODRIGUEZ JA, VRANIC M, RAMAIAH V, RAVI R, DIETHRICH EB. A prospective study of ultrasound scan-guided thrombin injection of femoral pseudoaneurysm: a trend toward minimal medication. Journal of Vascular Surgery 2002; 36: 779-82.

20. KANG SS, LABROPOULOS N, MANSOUR A, MICHELINI M, FILLIUNG D, BAUBLY MP, et al. Expanded indications for ultrasound-guided thrombin injection of pseudoaneurysms. J Vasc Surg 2000; 31: 289-98.

21. ALTIN RS, FLICKER S, NAIDECH HJ. Pseudoaneurysm and arteriovenous fistula after femoral artery catheterization: asociación with low femoral punctures. AJR Am J Roentgenol 1989; 152: 629-63

22. TAGNEY J, LACKIE D. Bed-rest post femoral arterial sheath removal: What is safe practice? A clinical audit. Nurs Crit Care 2005; 10: 167-173

23. THORÉ V, BERDER V, HOUPLON P, PREISS JP, SELTON-SUTY C, JUILLIÈRE Y. Role of manual compression time and bed rest duration on the occurrence of femoral bleeding complications after sheath retrieval following 4Fr left sided cardiac catheterization. J Interv Cardiol 2001; 14: 7- 10

24. KORENY M, RIEDMULLER E, NIKFARDJAM M, SIOSTRZONEK P, MÜLLNER M. Arterial puncture closing devices compared with standard manual compression after cardiac catheterization: systematic review and metaanalysis. JAMA 2004; 291: 350-357. 